(C) 2000 International Press

Adv. Theor. Math. Phys. 4 (2000) 775-790

\title{
Membranes on Fivebranes
}

\author{
Jerome P. Gauntlett
}

\author{
Department of Physics \\ Queen Mary and Westfield College \\ University of London \\ Mile End Road \\ London E1 4NS, UK \\ J.P.Gauntlett@qmw.ac.uk
}

\begin{abstract}
By analysing supersymmetry transformations we derive new BPS equations for the $D=11$ fivebrane propagating in flat space that involve the world-volume three-form. The equations generalise those of 2,3,4 and 5 dimensional special Lagrangian submanifolds and are relevant for describing membranes ending on these submanifolds.
\end{abstract}

e-print archive: http://xxx.lanl.gov/hep-th/9906162 


\section{Introduction}

The world-volume theories of branes in string theory and M-theory are an interesting setting for studying BPS equations. One notable feature is that the solutions incorporate a spacetime interpretation $[8$, 14, 18] which arises from the fact that the scalar fields describe the embedding of the brane in a target space. The world-volume theory of the $D=11$ fivebrane, for example, has a BPS self-dual string soliton that preserves $1 / 2$ of the world-volume supersymmetry, or equivalently, $1 / 4$ of the spacetime supersymmetry [18]. It is charged with respect to the world-volume self-dual three-form and one scalar field is excited that is harmonic in four variables. The simplest solution with a single centre can then be visualised as a semi-infinite membrane ending on the fivebrane. This configuration can be represented as the array

$$
\begin{array}{lllllll}
M 5: & 1 & 2 & 3 & 4 & 5 & \\
M 2: & 1 & & & & & 6
\end{array}
$$

with the self-dual string lying in the 1 direction and the 6 direction corresponding to the excited scalar field.

Here we will derive the BPS equations corresponding to adding more membranes to this array. Specifically, we will analyse arrays with $2,3,4$ and 5 orthogonally intersecting membranes, lying in the $\{2,7\},\{3,8\}$, $\{4,9\}$ and $\{5,10\}$ directions, which preserve $1 / 8,1 / 16,1 / 32$ and $1 / 32$ of the spacetime supersymmetry, respectively. The BPS equations are obtained by analysing the supersymmetry transformations of the fivebrane world-volume theory in a flat target space. As in $[10,11]$, the array of orthogonally intersecting branes provides a guide in constructing the BPS equations. More specifically, each membrane in the array of intersecting branes suggests which projections to impose on the supersymmetry spinor parameters and in addition which scalar fields should be active. This information then leads to the BPS equations.

We will argue that the equations are appropriate for describing a membrane ending on a fivebrane world-volume, some of which is a special Lagrangian submanifold. To see this, we first note that a consequence of the projections imposed on the spinor parameters arising from the arrays of membranes orthogonally intersecting a single fivebrane, is that one can add certain fivebranes to the array "for free", i.e., without imposing further conditions on the spinor parameters. Following 
$[4,5]$, it was shown in [10] (see also $[15,1]$ ) that an array of intersecting fivebranes only corresponds to BPS equations on the world-volume of a single fivebrane that imply that the fivebrane world-volume (or part of it) is a calibrated submanifold [16]. By adding extra membranes to the array (1) we find that the fivebranes we can add for free correspond to the special Lagrangian calibrations. More precisely, adding 2,3,4 and 5 membranes corresponds to the projections for fivebranes related to 2,3,4 and 5 dimensional special Lagrangian manifolds, respectively. The full BPS equations will involve the world-volume three-form and can be interpreted as membranes ending on such fivebrane world-volumes ${ }^{1}$. It should be emphasised that since the BPS equations incorporate back reaction, the fivebrane world-volume will in general no longer be special Lagrangian. Both the geometry and the topology could be different.

Let us illustrate this interpretation further for the simplest case. As we will later see explicitly, adding a membrane in the $\{2,7\}$ directions to the array (1) gives rise to an extra projection on the supersymmetry parameters which implies that we can add an anti-fivebrane in the $\{3,4,5,6,7\}$ directions for free to obtain the array:

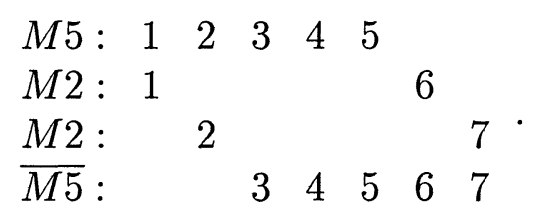

The two intersecting fivebranes alone correspond to a two-dimensional special Lagrangian submanifold, or equivalently, a complex curve in the $\{1,2,6,7\}$ directions. This is the M-theory setup that has been used to analyse $N=2$ superYang-Mills (SYM) theory [23]. By including the membranes, the corresponding BPS equations will have solutions that can be interpreted as a membrane ending on this curve. In the Yang-Mills setting these configurations will correspond to monopoles and dyons. Such membranes were first considered in [9, 17, 22] without including the back-reaction on the fivebrane. The BPS equations that we discuss here which do include the back-reaction were first presented in $[11,21]$ and the application to dyons was analysed in [21]. In [11, 21] the BPS equations were derived and checked in a certain approximation in the "covariant formalism" of the fivebrane [19]. Here we shall

\footnotetext{
${ }^{1}$ Note that BPS equations for other configurations of intersecting fivebranes and membranes were derived in [11].
} 
derive the exact BPS equations in the Hamiltonian formalism [6]. A consequence of this is that we will be able to present the exact energy functional for these configurations that was presented in approximate form in [3].

After discussing this case we will consider arrays with three intersecting membranes. We will see that the projections for the membranes imply the projections corresponding to a three dimensional special Lagrangian submanifold. Such submanifolds are relevant for describing $N=2 \mathrm{SYM}$ in $d=3[20]$ and the equations for the membranes correspond to solitonic excitations in these theories. The BPS equations that we derive provide a starting point to analyse such states.

The projections for four intersecting membranes automatically imply those for five so we will consider these cases together. The resulting BPS equations are appropriate for studying membranes ending on four or five dimensional special Lagrangian submanifolds. The BPS equations for the five membrane case are in fact the most general in the sense that by setting certain scalar fields to zero we can recover the BPS equations for all previous cases. In all cases the equations are rather complicated and (almost) no attempt will be made for finding solutions here.

\section{Two Intersecting Membranes}

To derive the BPS equations we will use the Hamiltonian formalism of the fivebrane theory [6] which is derived from the "Lagrangian" formalism of [2]. The bosonic world volume fields consist of scalars $X^{\mu}$, $\mu=0, \ldots, 10$, and a closed three form field-strength $H$ that satisfies a self-duality condition. In the static gauge we have $X^{0}=\sigma^{0}$, $X^{a}=\sigma^{a}, a, b, \cdots=1, \ldots, 5$, where $\left(\sigma^{0}, \sigma^{a}\right)$ are coordinates on the fivebrane world-volume. We will only consider static, bosonic configurations $^{2} X^{T}\left(\sigma^{a}\right), T=6, \ldots, 10, H_{a b c}\left(\sigma^{a}\right)$, and we shall take a flat target space. The spatial components of the world-volume metric, $g_{a b}$,

\footnotetext{
${ }^{2}$ In the Hamiltonian formalism we only need to specify the spatial components of $H$.
} 
are then given by

$$
g_{a b}=\delta_{a b}+\partial_{a} X^{T} \partial_{b} X^{T}
$$

It was shown in [11] that bosonic configurations that preserve supersymmetry must satisfy

$$
\sqrt{\operatorname{det}(g+\tilde{H})_{a b}}=\epsilon^{\dagger} \gamma^{0}\left[\frac{1}{5 !} \Gamma_{a_{1} \ldots a_{5}} \epsilon^{a_{1} \ldots a_{5}}-\frac{\sqrt{g}}{2} \Gamma_{a b} \tilde{H}^{a b}+\Gamma_{a} t^{a}\right] \epsilon
$$

where

$$
\begin{aligned}
\tilde{H}^{a b} & =\frac{1}{3 !} \frac{1}{\sqrt{g}} \varepsilon^{a b c_{1} c_{2} c_{3}} H_{c_{1} c_{2} c_{3}} \\
t_{f} & =\frac{1}{4 !} \varepsilon^{a b c d e} H_{a b c} H_{d e f}
\end{aligned}
$$

and $\epsilon$ is a 32 component Majorana spinor satisfying $\epsilon^{\dagger} \epsilon=1$. We use the convention that $\varepsilon^{12345}=1$. The gamma-matrices $\Gamma_{a}$ are the flat spacetime matrices, $\gamma_{\mu}$, pulled back to the world-volume:

$$
\Gamma_{a}=\gamma_{a}+\partial_{a} X^{T} \gamma_{T}
$$

It is useful to introduce the density

$$
\tilde{\mathcal{H}}^{a b}=\sqrt{g} \tilde{H}^{a b}
$$

so that the closure of the three-form $H$ is then $\partial_{a} \tilde{\mathcal{H}}^{a b}=0$.

We now turn to the arrays of two membranes intersecting a fivebrane according to the pattern (2). To preserve supersymmetry we must impose the following projections on the supersymmetry parameters:

$$
\begin{aligned}
\gamma^{012345} \epsilon & =\epsilon \\
\gamma^{016} \epsilon & =\epsilon \\
\gamma^{027} \epsilon & =\epsilon
\end{aligned}
$$

and hence the configuration preserves $1 / 8$ of the supersymmetry. These projections imply that $\gamma^{034567} \epsilon=-\epsilon$ which means that we can indeed include the final fivebrane in (2) for free, as claimed earlier. To realise this configuration of intersecting branes as a supersymmetric configuration of the first fivebrane we set all scalar fields to zero except for 
$X^{6} \equiv Y^{1}$ and $X^{7} \equiv Y^{2}$, which will be functions of $\sigma^{a}=\left(\sigma^{i}, \sigma^{\alpha}\right)$, where, in this section, $i, j, \cdots=1,2$ and $\alpha, \beta, \cdots=3,4,5$. By substituting this ansatz into (4) and imposing the projections (8) we will be able to derive the BPS equations.

It will be convenient to introduce the notation $\left(\rho^{1}, \rho^{2}\right)=\left(\gamma^{6}, \gamma^{7}\right)$, so that projections on the spinor imply that

$$
\begin{aligned}
& \gamma_{1} \rho_{1} \epsilon=\gamma_{2} \rho_{2} \epsilon=\gamma_{12345} \epsilon \\
& \gamma_{1} \rho_{2} \epsilon=-\gamma_{2} \rho_{1} \epsilon .
\end{aligned}
$$

We then find that

$$
\begin{aligned}
\Gamma_{12345} \epsilon & =\left[\left(1-\operatorname{det} \partial_{i} Y^{j}\right) \gamma_{12345}+\partial_{i} Y^{i}+\partial_{i} Y^{j} \epsilon_{i j} \gamma_{12}+\partial_{\alpha} Y^{i} \gamma_{\alpha i}\right. \\
& \left.+\left(\partial_{\alpha} Y^{j} \partial_{j} Y^{i}-\partial_{\alpha} Y^{i} \partial_{j} Y^{j}\right) \gamma_{\alpha} \rho_{i}+\epsilon_{\alpha \beta \gamma} \partial_{\beta} Y^{1} \partial_{\gamma} Y^{2} \gamma_{\alpha}\right] \epsilon
\end{aligned}
$$

Similarly

$$
\begin{aligned}
-\frac{1}{2} \tilde{\mathcal{H}}^{a b} \Gamma_{a b} \epsilon= & {\left[\left(\tilde{\mathcal{H}}^{12} \epsilon_{i j} \partial_{i} Y^{j}+\tilde{\mathcal{H}}^{\alpha i} \partial_{\alpha} Y^{i}\right) \gamma_{12345}\right.} \\
& +\left(\partial_{\alpha} Y^{k} \tilde{\mathcal{H}}^{\alpha i} \partial_{i} Y^{l} \epsilon_{k l}-\tilde{\mathcal{H}}^{12}\left(1-\operatorname{det} \partial_{i} Y^{j}\right)\right) \gamma_{12} \\
& -\tilde{\mathcal{H}}^{\alpha i} \gamma_{\alpha i}-\tilde{\mathcal{H}}^{\alpha j} \partial_{j} Y^{i} \gamma_{\alpha} \rho_{i} \\
& \left.+\left(\tilde{\mathcal{H}}^{12} \partial_{i} Y^{i}-\tilde{\mathcal{H}}^{\alpha[i} \partial_{\alpha} Y^{j]} \epsilon_{i j}\right) \gamma_{2} \rho_{1}-\tilde{\mathcal{H}}^{\alpha \beta} \Gamma_{\alpha \beta}\right] \epsilon
\end{aligned}
$$

and

$$
t^{a} \Gamma_{a} \epsilon=t^{a} \gamma_{a} \epsilon+t^{a} \partial_{a} Y^{i} \rho_{i} \epsilon
$$

To ensure the equality of the two sides of (4) the sum of (10)-(12) must vanish except for terms proportional to $\gamma_{12345} \epsilon$. We conclude that

$$
\begin{aligned}
\tilde{\mathcal{H}}^{\alpha \beta} & =0 \\
\tilde{\mathcal{H}}^{\alpha i} & =\partial_{\alpha} Y^{i} \\
\tilde{\mathcal{H}}^{12} & =\frac{\epsilon_{i j} \partial_{i} Y^{j}+\partial_{\alpha} Y^{k} \partial_{\alpha} Y^{i} \partial_{i} Y^{l} \epsilon_{k l}}{1-\operatorname{det} \partial_{i} Y^{j}} \\
\partial_{i} Y^{i} & =0 .
\end{aligned}
$$

The first equation comes from noting that $\Gamma_{\alpha \beta}=\gamma_{\alpha \beta}+\ldots$ and ensuring that the $\gamma_{\alpha \beta} \epsilon$ terms vanish. The next three equations follow from the vanishing of the terms $\gamma_{\alpha i} \epsilon, \gamma_{12} \epsilon$ and $\epsilon$, respectively. Using these it 
is straightforward to show that the $\gamma_{\alpha} \rho_{i} \epsilon$ and $\gamma_{2} \rho_{1} \epsilon$ terms also vanish. These equations also imply that

$$
\begin{aligned}
t_{\alpha} & =-\epsilon_{\alpha \beta \gamma} \partial_{\beta} Y^{1} \partial_{\gamma} Y^{2} \\
t_{i} & =0
\end{aligned}
$$

From the functional form of $g^{a b}$ we then conclude that $t^{a}=g^{a b} t_{b}=t_{a}$. These facts then ensure the vanishing of the $\gamma_{\alpha} \epsilon, \gamma_{i} \epsilon$ and $\rho_{i} \epsilon$ terms.

To match the two sides of (4) we are thus left to show that

$$
\sqrt{\operatorname{det}(g+\tilde{H})}=1-\operatorname{det} \partial_{i} Y^{j}+\partial_{\alpha} Y^{i} \partial_{\alpha} Y^{i}+\tilde{\mathcal{H}}^{12}\left(\partial_{1} Y^{2}-\partial_{2} Y^{1}\right) .
$$

We first note that the determinant can be rewritten

$$
\operatorname{det}\left(g_{a b}+\tilde{H}_{a b}\right)=g+\frac{1}{2} \tilde{\mathcal{H}}^{a b} \tilde{\mathcal{H}}_{a b}+g^{a b} t_{a} t_{b} .
$$

and using (14) we have

$$
g^{a b} t_{a} t_{b}=\left(\nabla Y^{1} \times \nabla Y^{2}\right)^{2},
$$

where $\nabla=\left(\partial_{3}, \partial_{4}, \partial_{5}\right)$. We can then use Mathematica to show using (13) that (15) is indeed an identity. The closure of $H$ imposes one additional equation:

$$
\partial_{\alpha} \partial_{\alpha} Y^{i}=\epsilon_{i j} \partial_{j} \tilde{\mathcal{H}}^{12}
$$

In summary, we have shown that (13), (18) are the relevant BPS equations corresponding to the configuration of intersecting branes in (2), and that the solutions will preserve $1 / 8$ of the spacetime supersymmetry. These BPS equations were first presented in $[11,21]$ using the covariant formalism of the fivebrane [19]. The preservation of supersymmetry was checked up to terms quadratic in the spatial derivatives $\partial_{\alpha}$. By contrast here we have checked the supersymmetry in the Hamiltonian formalism exactly.

The form of $\tilde{\mathcal{H}}^{\alpha 1}$ in the BPS equations is somewhat analogous to that of a membrane in the $\{0,1,6\}$ directions and $\tilde{\mathcal{H}}^{\alpha 2}$ that of a membrane in the $\{0,2,7\}$ directions. More specifically, if we set $Y^{2}=0$, say, then we precisely obtain the BPS equations for a single self-dual string in the 1 direction. Alternatively, if one sets $\partial_{\alpha}=0$ and imposes 
$\partial_{1} Y^{2}=\partial_{2} Y^{1}$ we get $H=0$ and combined with the last equation in (13) we are left with the Cauchy-Riemann equations for $Y^{1}, Y^{2}$ as a function of $\sigma^{1}, \sigma^{2}$, corresponding to the two intersecting fivebranes. We thus conclude that the equations generalise the Cauchy-Riemann equations to include a non-zero $H$ and thus are relevant for describing membranes ending on complex curves in four dimensions, or equivalently, a two dimensional special Lagrangian submanifold. As the equations include the back-reaction of the membrane on the fivebrane geometry, the fivebrane world-volume will in general no longer be a complex curve in the $\{1,2,6,7\}$ directions because only half of the Cauchy-Riemann equations are imposed. Note that one can impose $\partial_{1} Y^{2}=\partial_{2} Y^{1}$ and still have non-zero $H$ if $\partial_{\alpha} \neq 0$. These equations are somewhat simpler, but it was shown in [21] that they do not admit finite energy solutions, and hence only provide an asymptotic description of the dyons in N=2 SYM theory. Finally, if one imposes $\partial_{i}=0$ we obtain the BPS equations for the delocalised intersecting self-dual strings discussed in [10] and [13].

We conclude this section with a comment about the energy of solutions solving (13). It was shown in [12] that the energy density squared of static fivebrane configurations is given by

$$
\mathcal{E}^{2}=\operatorname{det}\left(g_{a b}+\tilde{H}_{a b}\right)+t_{a} t_{b} m^{a b}
$$

with $m^{a b}=\delta^{a b}-g^{a b}$. For the configurations we have been considering we have $t_{a} t_{b} m^{a b}=0$ and hence

$$
\begin{aligned}
\mathcal{E} & =\sqrt{\operatorname{det}\left(g_{a b}+\tilde{H}_{a b}\right)} \\
& =\frac{\operatorname{det} g-\left(\nabla Y^{1} \times \nabla Y^{2}\right)^{2}}{1-\operatorname{det} \partial_{i} Y^{j}} .
\end{aligned}
$$

This last expression differs from that given in [3] but agrees with it up to terms quadratic in the spatial derivatives which was the approximation employed there. Note that if we impose the other Cauchy-Riemann equation on $Y^{1}$ and $Y^{2}, \partial_{1} Y^{2}=\partial_{2} Y^{1}$, we obtain

$$
\mathcal{E}=1+\partial_{j} Y^{i} \partial_{j} Y^{i}+\partial_{\alpha} Y^{i} \partial_{\alpha} Y^{i}
$$

as in [3]. 


\section{$3 \quad$ Three Intersecting Membranes}

We now consider adding another membrane to the array (2) to obtain

$\begin{array}{lllllllll}M 5: & 1 & 2 & 3 & 4 & 5 & & & \\ M 2: & 1 & & & & & 6 & & \\ M 2: & & 2 & & & & & 7 & \\ M 2: & & & 3 & & & & & 8\end{array}$.

To preserve supersymmetry we must now impose $\gamma^{038} \epsilon=\epsilon$ in addition to (8). This means that the configuration preserves $1 / 16$ of the spacetime supersymmetry. If we denote $\left(\gamma^{6}, \gamma^{7}, \gamma^{8}\right) \equiv\left(\rho^{1}, \rho^{2}, \rho^{3}\right)$ then we have

$$
\begin{aligned}
& \gamma^{i} \rho^{i} \epsilon=\gamma^{1} \rho^{1} \epsilon=\gamma_{12345} \epsilon \quad \text { no sum on } i \\
& \gamma^{i} \rho^{j} \epsilon=-\gamma^{j} \rho^{i} \epsilon \quad i \neq j,
\end{aligned}
$$

where in this section $i, j, \cdots=1,2,3$. As in the previous section these projections imply that we can add several other fivebranes for free. In fact one finds we can add the fivebranes corresponding to three dimensional special Lagrangian submanifolds (see section 2.2 of [10]).

To realise the intersection of such membranes and fivebranes on the first fivebrane we let three scalars be active: $\left(X^{6}, X^{7}, X^{8}\right) \equiv\left(Y^{1}, Y^{2}, Y^{3}\right)$ with $Y^{i}=Y^{i}\left(\sigma^{j}, \sigma^{\alpha}\right)$, where in this section $\alpha, \beta, \cdots=4,5$. As before we substitute this into (4) and impose the projections to determine $\tilde{\mathcal{H}}$. The vanishing of the terms with $\epsilon, \gamma_{\alpha \beta} \epsilon, \gamma_{\alpha i} \epsilon$ and $\gamma_{i j} \epsilon$ imply, respectively, that

$$
\begin{aligned}
\partial_{i} Y^{i} & =\operatorname{det}\left(\partial_{i} Y^{j}\right) \\
\tilde{\mathcal{H}}^{\alpha \beta} & =0 \\
\tilde{\mathcal{H}}^{\alpha i} & =M_{i j} \partial_{\alpha} Y^{j} \\
\tilde{\mathcal{H}}^{i j} & =\epsilon^{i j k} A^{k} \\
A^{l} & =\left(\partial_{i} Y^{j}+\partial_{\alpha} Y^{m} \partial_{\alpha} Y^{i} \partial_{m} Y^{j}\right) \epsilon_{i j k} M_{k l}^{-1}
\end{aligned}
$$

where the matrix $M$ is defined as

$$
M_{i j}=\delta_{i j}-(-1)^{i+j} \operatorname{det}_{i \mid j}\left(\partial_{k} Y^{l}\right)
$$

where the subscript on the $\operatorname{det}_{i \mid j}$ indicates that we remove the $i$ th row and $j$ th column of the matrix $\partial_{k} Y^{l}$ before taking the determinant. It 
is then straightforward to show that the $\gamma_{\alpha} \rho_{i} \epsilon$ and $\gamma_{[i} \rho_{j]} \epsilon$ terms automatically vanish using the lemmas

$$
\begin{aligned}
\tilde{\mathcal{H}}^{\alpha i} \partial_{i} Y^{j} & =\partial_{\alpha} Y^{i} \partial_{i} Y^{j}-\partial_{\alpha} Y^{j}\left(\partial_{l} Y^{l}\right) \\
M_{i j} \partial_{k} Y_{j} & =\partial_{k} Y^{i}-\left(\partial_{l} Y^{l}\right) \delta_{i k}
\end{aligned}
$$

One can use Mathematica to show that (24) implies

$$
\begin{aligned}
t^{k} & =-\frac{1}{2} \epsilon_{\alpha \beta} \partial_{\alpha} Y^{i} \partial_{\beta} Y^{j} \epsilon_{i j k} \\
t^{\alpha} & =\epsilon_{\alpha \beta} \partial_{\beta} Y^{i} \partial_{j} Y^{k} \epsilon_{i j k}
\end{aligned}
$$

and hence the vanishing of the $\gamma^{k} \epsilon$ and $\gamma^{\alpha} \epsilon$ terms. By expanding the identity

$$
\partial_{\alpha} Y^{[i} \partial_{\beta} Y^{j} \partial^{k} Y^{l]}=0
$$

one can then show that

$$
t^{i} \partial_{i} Y^{l}+t^{\alpha} \partial_{\alpha} Y^{l}=-\frac{1}{2} \epsilon_{\alpha \beta} \partial_{\alpha} Y^{i} \partial_{\beta} Y^{j} \epsilon_{i j k} \partial_{k} Y^{l}
$$

and hence the $\rho_{i} \epsilon$ terms vanish. To complete the proof of supersymmetry one is then left to prove that

$$
\sqrt{\operatorname{det}(g+\tilde{H})}=1-\frac{1}{2}\left(\partial_{i} Y^{i}\right)^{2}+\frac{1}{2} \partial_{i} Y^{j} \partial_{j} Y^{i}+\tilde{\mathcal{H}}^{\alpha i} \partial_{\alpha} Y^{i}+\tilde{\mathcal{H}}^{i j} \partial_{i} Y^{j}
$$

This can again be established using Mathematica. The closure of the three form $H$ then implies that

$$
\partial_{\alpha} \tilde{\mathcal{H}}^{\alpha j}+\partial_{i} \tilde{\mathcal{H}}^{i j}=0
$$

The other equation, $\partial_{a} \tilde{\mathcal{H}}^{a \beta}=0$, is automatically satisfied by virtue of (24).

Let us discuss some special cases of these equations. Firstly we note that if we impose

$$
\partial_{i} Y^{j}=\partial_{j} Y^{i}, \quad \partial_{\alpha} Y^{i}=0
$$

then we have $\tilde{\mathcal{H}}=0$ and the equations are simply those of a three dimensional special Lagrangian submanifold [16]. The more general 
equations can thus be seen to include cases relevant for describing membranes ending on such submanifolds. Note that we can just impose the first equation in (32) leading to the equations for a three dimensional special Lagrangian manifold with non-zero three-form. By analogy with the case of two intersecting membranes one is lead to suspect that this simplification will not admit finite energy solutions.

We next consider the case where $Y^{i}$ is a function of $\sigma^{\alpha}$ only. In this case the self-dual strings are delocalised in the $\sigma^{i}$ directions. In this case we find

$$
\begin{aligned}
\tilde{\mathcal{H}}^{\alpha i} & =\partial_{\alpha} Y^{i} \\
\tilde{\mathcal{H}}^{i j} & =\tilde{\mathcal{H}}^{\alpha \beta}=0
\end{aligned}
$$

and the closure of $H$ then implies that the $Y^{i}$ are harmonic functions of two variables. The solutions of these delocalised membranes then have a logarithmic behaviour. Note that the energy of these solutions is

$$
\mathcal{E}=1+\partial_{\alpha} Y^{i} \partial_{\alpha} Y^{i}
$$

Finally, if we set $Y^{3}=0$ then the equations become exactly those of the last section corresponding to two intersecting self-dual strings.

\section{Four and Five Intersecting Membranes}

The projections imposed on the spinor parameters for the case of four intersecting membranes with a fivebrane automatically imply the projections for a fifth membrane so we shall analyse these cases together. The general configuration can be written

$\begin{array}{lllllllllll}M 5: & 1 & 2 & 3 & 4 & 5 & & & & \\ M 2: & 1 & & & & & 6 & & & \\ M 2: & & 2 & & & & & 7 & & & \\ M 2: & & & 3 & & & & & 8 & & \\ M 2: & & & 4 & & & & & 9 & \\ M 2: & & & & 5 & & & & & \end{array}$.


where $\sharp$ represents the number 10 . In addition to (8) we must impose $\gamma^{038} \epsilon=\epsilon, \gamma^{049} \epsilon=\epsilon \gamma^{05 \sharp} \epsilon=\epsilon$. We then have:

$$
\begin{aligned}
& \gamma^{a} \rho^{a} \epsilon=\gamma^{1} \rho^{1} \epsilon=\gamma_{12345} \epsilon \quad \text { no sum on } a \\
& \gamma^{a} \rho^{b} \epsilon=-\gamma^{b} \rho^{a} \epsilon \quad a \neq b
\end{aligned}
$$

where $\left(\rho^{1}, \ldots, \rho^{5}\right) \equiv\left(\gamma^{6}, \ldots, \gamma^{\sharp}\right)$ and $a, b, \cdots=1, \ldots, 5$. These projections are those for five dimensional special Lagrangian submanifolds; the corresponding fivebranes that one can add to (35) for free can be found in section 2.4 of [10]. For this case all five scalar fields $\left(X^{6}, \ldots, X^{\sharp}\right) \equiv\left(Y^{1}, \ldots, Y^{5}\right)$ are excited. Again we substitute into (4) and demand equality. The right hand side of (4) becomes

$$
\begin{aligned}
& \left(1-\frac{1}{2}\left(\partial_{a} Y^{a}\right)^{2}+\frac{1}{2} \partial_{a} Y^{b} \partial_{b} Y^{a}+\underset{a \mid a}{\operatorname{det}} \partial Y+\tilde{\mathcal{H}}^{a b} \partial_{a} Y^{b}\right) \\
& +\left(\partial_{a} Y^{a}-\frac{1}{2} \Sigma_{a \neq b} \operatorname{det}_{a b \mid a b} \partial Y+\operatorname{det} \partial Y\right) \epsilon^{\dagger} \gamma^{0} \epsilon \\
& +\left(t^{e}+\frac{1}{2} \partial_{a} Y^{c} \partial_{b} Y^{d} \epsilon^{a b c d e}\right) \epsilon^{\dagger} \gamma^{0 e} \epsilon \\
& +\left(\partial_{[c} Y_{d]}+3 \partial_{[a} Y^{c} \partial_{b} Y^{a} \partial_{d]} Y^{b}-\frac{1}{2} \tilde{\mathcal{H}}^{c d}+\frac{1}{2} \tilde{\mathcal{H}}^{a b} \partial_{a} Y^{[c} \partial_{b} Y^{d]}\right) \epsilon^{\dagger} \gamma^{0 c d} \epsilon \\
& +\left(\tilde{\mathcal{H}}^{a[b} \partial_{a} Y^{c]}-\partial_{a} Y^{a} \partial_{[b} Y_{c]}+\partial_{[b} Y^{a} \partial_{|a|} Y_{c]}\right. \\
& \left.\quad+(-1)^{b+c} \operatorname{det}_{b \mid c} \partial Y\right) \epsilon^{\dagger} \gamma^{0} \gamma_{[b} \rho_{c]} \epsilon \\
& +\left(t^{a} \partial_{a} Y^{e}+\frac{1}{2} \epsilon_{b_{1} b_{2} b_{3} b_{4} b_{5}} \partial_{b_{4}} Y^{b_{1}} \partial_{b_{5}} Y^{b_{2}} \partial_{e} Y^{b_{3}}\right) \epsilon^{\dagger} \gamma^{0} \rho_{e} \epsilon
\end{aligned}
$$

where $Y_{i} \equiv Y^{i}$.

The vanishing of the $\epsilon^{\dagger} \gamma^{0} \epsilon$ terms implies the BPS equation

$$
\partial_{a} Y^{a}-\frac{1}{2} \Sigma_{a \neq b} \operatorname{det}_{a b \mid a b} \partial Y+\operatorname{det} \partial Y=0
$$

We next note that the vanishing of the $\epsilon^{\dagger} \gamma^{0 a b} \epsilon$ and $\epsilon^{\dagger} \gamma^{0} \gamma_{[b} \rho_{c]} \epsilon$ terms imply that

$$
\begin{aligned}
\tilde{\mathcal{H}}^{c d}-\tilde{\mathcal{H}}^{a b} \partial_{a} Y^{c} \partial_{b} Y^{d}-2 \partial_{[c} Y_{d]} \\
-3 \partial_{[a} Y^{c} \partial_{b} Y^{a} \partial_{d]} Y^{b}+3 \partial_{[a} Y^{d} \partial_{b} Y^{a} \partial_{c]} Y^{b}=0 \\
\tilde{\mathcal{H}}^{a[b} \partial_{a} Y^{c]}-\partial_{a} Y^{a} \partial_{[b} Y_{c]}+\partial_{[b} Y^{a} \partial_{|a|} Y_{c]}+Z_{b c}=0
\end{aligned}
$$


where

$$
Z_{b c}=\frac{1}{2}(-1)^{b+c}\left(\operatorname{det}_{b \mid c} \partial Y-\operatorname{det}_{c \mid b} \partial Y\right)
$$

If we multiply the second by $\partial_{b} Y^{d}$ and substitute into the first equation we find after some algebra

$$
\tilde{\mathcal{H}}^{a b}=T_{a c} N_{c b}^{-1}
$$

where

$$
\begin{aligned}
N_{a b}= & \delta_{a b}+\partial_{a} Y^{c} \partial_{c} Y^{d} \\
T_{a b}= & \left(\partial_{a} Y^{b}-\partial_{b} Y^{a}\right)\left(1-\frac{1}{2}\left(\partial_{c} Y^{c}\right)^{2}+\frac{1}{2} \partial_{c} Y^{d} \partial_{d} Y^{c}\right) \\
& +\left(\partial_{d} Y^{c} \partial_{c} Y^{a}-\left(\partial_{c} Y^{c}\right) \partial_{d} Y^{a}\right)\left(\partial_{b} Y^{d}-\partial_{d} Y^{b}\right)-2 Z_{c a} \partial_{c} Y^{b}
\end{aligned}
$$

We then need

$$
t^{e}=\frac{1}{2} \epsilon^{a b c d e} \partial_{a} Y_{b} \partial_{c} Y_{d}
$$

for the $\epsilon^{\dagger} \gamma^{0 a} \epsilon$ terms to vanish. Using the identity

$$
\epsilon^{a b c d e} \partial_{a} Y_{b} \partial_{c} Y_{d} \partial_{e} Y_{f}=\epsilon^{a b c d e} \partial_{a} Y_{b} \partial_{c} Y_{d} \partial_{f} Y_{e},
$$

which comes from the identity $\partial_{[a} Y_{b} \partial_{c} Y_{d} \partial_{e} Y_{f]}=0$, we conclude from (43) that the $\epsilon^{\dagger} \gamma^{0} \rho_{a} \epsilon$ terms also vanish.

We have thus shown that solutions to the BPS equations (38) and (41) together with the closure of $H, \partial_{a} \tilde{\mathcal{H}}^{a b}=0$, preserve $1 / 32$ of the supersymmetry provided that

$$
\sqrt{\operatorname{det}(g+\tilde{H})}=1-\frac{1}{2}\left(\partial_{a} Y^{a}\right)^{2}+\frac{1}{2} \partial_{a} Y^{b} \partial_{b} Y^{a}+\operatorname{det}_{a \mid a} \partial Y+\tilde{\mathcal{H}}^{a b} \partial_{a} Y^{b}
$$

We have not quite been able to show this with Mathematica using the machines available. However, we have made some highly non-trivial checks and we strongly expect that it is in fact true.

Note that if $\partial_{a} Y^{b}=\partial_{b} Y^{a}$ we have $\tilde{\mathcal{H}}^{a b}=0$ and the BPS equations truncate to those of a five dimensional special Lagrangian submanifold [16]. We note also that setting $Y^{5}=0$ will give rise to the BPS 
equations for four intersecting membranes associated with a four dimensional special Lagrangian submanifold, while if in addition we set $Y^{4}=0$ we recover the equations obtained in the last section. Thus, these BPS equations are the most general of the type considered in this paper.

\section{Conclusions}

We have constructed BPS equations corresponding to membranes ending on fivebranes and revealed how they generalise those of special Lagrangian submanifolds. The only new solutions that were presented here were those corresponding to three delocalised intersecting membranes. It would be very interesting to construct less trivial solutions, but the analysis presented in [21] for two intersecting membranes indicates that this could be very difficult.

If we dimensionally reduce the general configuration (35) on one of the fivebrane directions then we obtain a D-4-brane intersecting a fundamental string with four D-2-branes. By reducing the BPS equations that we have derived one will obtain BPS equations for the abelian Dirac-Born-Infeld theory relevant for these configurations. By additional T- and S-duality transformations, one can in principle obtain BPS equations for many different configurations of intersecting branes. Following [12] it was shown in [7] that simpler BPS equations for the abelian Dirac-Born-Infeld theory have a natural analogue for the nonabelian theory. Presumably this will also be true for these more complicated cases.

\section{Acknowledgements}

I would like to thank Fay Dowker, Jose Figueora-O'Farrill and Bill Spence for discussions and the EPSRC for partial support. 


\section{References}

[1] B.S. Acharya, J.M. Figueroa-O'Farrill, and B. Spence, J. High Energy Phys., 04 (1998), 012; hep-th/9803260.

[2] I. Bandos, K. Lechner, A. Nurmagambetov, P. Pasti, D. Sorokin, and M. Tonin, Phys. Lett., B408 (1997), 135; hep-th/9703127.

[3] O. Bärwald, N.D. Lambert, and P.C. West, On the Energy Momentum Tensor of the M Theory Fivebrane, hep-th/9904097.

[4] K. Becker, M. Becker, and A. Strominger, Nucl. Phys., B456 (1995), 130.

[5] K. Becker, M. Becker, D. Morrison, H. Ooguri, Y. Oz, and Z. Yin, Nucl. Phys., B480 (1996), 225; hep-th/9608116.

[6] E. Bergshoeff, D. Sorokin, and P.K. Townsend, Nucl.Phys., B533 (1998), 303; hep-th/9805065.

[7] D. Brecher, Phys. Lett., B442 (1998), 117.

[8] C.G. Callan Jr. and J.M. Maldacena, Nucl. Phys., B513 (1998), 198; hep-th/9708147.

[9] A. Fayyazuddin and M. Spalinski, Nucl. Phys., B508 (1997), 219; hep-th/9706087.

[10] J.P. Gauntlett, N.D. Lambert, and P.C. West, Commun. Math. Phys., 202 (1999), 571; hep-th/9803216.

[11] J.P. Gauntlett, N.D. Lambert and P.C. West, Adv. Theor. Math. Phys., 3 (1999) 91, hep-th/9811024.

[12] J.P. Gauntlett, J. Gomis, and P.K. Townsend, J. High Energy Phys., 01 (1998), 003, hep-th/9711025.

[13] J.P. Gauntlett, C. Koehl, D. Mateos, P. Townsend, and M. Zamaklar, Phys. Rev., D60 (1999), 045004, hep-th/9903156.

[14] G.W. Gibbons, Nucl. Phys., B514 (1998), 603; hep-th/9709027.

[15] G.W. Gibbons and G. Papadopoulos, Commun. Math. Phys., 202 (1999), 593; hep-th/9803163. 
[16] R. Harvey and H.B. Lawson, Jr., Acta Math., 148 (1982), 47.

[17] M. Henningson and P. Yi, Phys. Rev., D57 (1998), 1291; hep-th/9707251.

[18] P.S. Howe, N.D. Lambert, and P.C. West, Nucl. Phys., B515 (1998), 203; hep-th/9709014.

[19] P.S. Howe and E. Sezgin, Phys. Lett., B394 (1997), 62; hep-th/9611008;

P.S. Howe, E. Sezgin, and P.C. West, Phys. Lett., B399 (1997), 49; hep-th/9702008.

[20] A. Karch, D. Lust, and A. Miemiec, $N=1$ Supersymmetric Gauge Theories and Supersymmetric 3-cycles, hep-th/9810254.

[21] N.D. Lambert and P.C. West, Monopole Dynamics from the $M$ Fivebrane, hep-th/9811025.

[22] A. Mikhailov, Nucl.Phys., B533 (1998), 243; hep-th/9708068.

[23] E. Witten, Nucl. Phys., B500 (1997), 3; hep-th/9703166. 